\title{
Performance of cassette-based blood gas analyzers to monitor blood glucose and lactate levels in a surgical intensive care setting
}

\begin{abstract}
Background: With the use of a traditional blood gas analyzer (BGA) (ABL800 Radiometer) for blood glucose monitoring, tight glucose control (TGC) reduced in-hospital mortality and morbidity of surgical intensive care unit (ICU) adult and pediatric patients. Such BGAs are now superseded by cassette-based BGAs. We assessed the analytical reliability of cassette-based BGAs to monitor patient's metabolic status in an ICU setting.

Methods: We evaluated recovery/linearity, imprecision/ repeatability and relative accuracy vs. comparison methods for glucose [coupled hexokinase glucose-6-phosphate dehydrogenase (HK/G6PD) assay] and lactate (lactate dehydrogenase assay) in ICU patient samples with two cassette-based BGAs [RP500 (Siemens) and ABL90 (Radiometer)] and with the ABL800 BGA.

Results: Recovery of spiked glucose up to $348 \mathrm{mg} / \mathrm{dL}$ (19.3 mmol/L) was complete for all BGAs. Repeatability of ABL800 and ABL90 was comparable with the comparison method (about 1\%), but higher for RP500 (about 2.4\%). All BGAs were in agreement with the comparison method, with all glucose measurements falling within preset $10 \%$ criteria suggested by Karon. Recovery of spiked lactate (up to $25 \mathrm{mmol} / \mathrm{L}$ ) was incomplete at all levels. Repeatability of ABL800 and ABL90 was comparable with the comparison method (about 4\%), and 5.5\% for RP500. All BGAs were in agreement with the comparison method, with $>98 \%$ of the lactate measurements falling within 30\% biologicalvariation-based criteria.

Conclusions: The cassette-based BGAs quantified blood glucose and lactate levels in ICU patients within the acceptable error ranges. Cassette-based BGAs can be used for monitoring patient's metabolic status in an ICU setting.
\end{abstract}

Keywords: blood gas analyzer; glucose; lactic acid; pointof-care systems; tight glucose control.
*Corresponding author: Florent Vanstapel, Laboratory Medicine, UZ Leuven, Campus Gasthuisberg, Herestraat 49, 3000 Leuven, Belgium, E-mail: florent.vanstapel@uzleuven.be Koen Poesen, Martine De Prins and Johan Van Eldere: Laboratory Medicine, UZ Leuven, Leuven, Belgium

Greet Van den Berghe: Intensive Care Medicine, UZ Leuven, Leuven, Belgium; and Department of Cellular and Molecular Medicine, Biomedical Sciences Group, KU Leuven, Belgium Johan Van Eldere: Laboratory of Clinical Bacteriology and Mycology, Department of Microbiology and Immunology, Biomedical Sciences Group, KU Leuven, Belgium

Florent Vanstapel: Department of Public Health, Biomedical Sciences Group, KU Leuven, Belgium

\section{Introduction}

Strict maintenance of normoglycemia [ $80-110 \mathrm{mg} / \mathrm{dL}$ (4.4-6.1 $\mathrm{mmol} / \mathrm{L})$ ] by intensive insulin therapy, termed tight glucose control (TGC), reduced in-hospital mortality and morbidity of surgical intensive care unit (ICU) adult and pediatric patients $[1,2]$. Later studies failed to confirm this benefit, sparking a continuing debate [3, 4]. Targeting a narrow $80-110 \mathrm{mg} / \mathrm{dL}(4.4-6.1 \mathrm{mmol} / \mathrm{L})$ range requires frequent (minimally every hour) and accurate quantification of the blood glucose concentration. The results need to be available within minutes for accurate and immediate insulin dose adaptations. Point-of-care measurement with the best available analytical technology is required [5].

The landmark studies that showed an improved outcome with TGC used a blood gas analyzer (BGA) embedded in an ICU environment to quantify blood glucose [1, 2]. The subsequent 'repeat' studies used handheld glucose meters [6]. It has become clear that the latter instruments do not have the accuracy required for targeting narrow blood glucose ranges [7-10]. Currently, BGAs are considered the only accurate point-of-care tools available for targeting narrow ranges of blood glucose in ICU patients [7].

Blood lactate is another metabolic parameter, used to diagnose tissue oxygen debt. As lactate measurements guide immediate clinical interventions as well, the analysis is also done with BGAs. 
The traditional BGAs used in the landmark studies on TGC [1] have individual electrodes for each measured parameter and separate bottles and vials for calibrators, quality control $(\mathrm{QC})$ materials, rinse solution and waste. The recent advances in BGA technology allow miniaturization of the reference electrode and working electrodes into one sensor cassette, reducing the complexity of technical interventions. Combining calibrators, reagents and QC material into one or more solution packs simplifies logistic interventions. The analytical reliability of BGAs, and of cassette-based BGAs in particular, to monitor metabolic status (glucose and lactate) of patients in an ICU setting has been scarcely investigated. In the present study we focused on the analytical performance of glucose and lactate measurements with ABL90 (Radiometer) and RP500 (Siemens) cassette systems as well as with a traditional BGA (ABL800, Radiometer) in an ICU setting.

\section{Materials and methods}

The study protocol was approved by the Institutional Ethical Review Board (ML7804). As blood sampling was performed in the context of standard clinical practice, the need for informed consent was waived.

Performance of glucose and lactate measurements was evaluated for the ABL800 and ABL90 (Radiometer Medical ApS, Brønshøj, Denmark) and RP500 (Siemens Healthcare, Erlangen, Germany) BGA. Summarized, glucose calibrators on all systems are traceable to NIST reference material (SRM 917a) and the coupled hexokinase glucose6-phosphate dehydrogenase (HK/G6PD) method [11-13]. For lactate, the Radiometer calibrator is traceable to lithium L-(+)-lactate (L-2250, Sigma Chemical Company), and the Siemens calibrator to an unspecified commercial material. Radiometer refers to a spectrophotometric method [14], whereas Siemens used a non-specified in-house method using lactate oxidase (ABL90 flex reference manual version 2.5xx and RL 1265 operator's manual version 06621382 Rev. S, 2007-07).

The comparison glucose assay on Roche/Hitachi Modular P analyzer (Roche Diagnostics, Mannheim, Germany) is a kinetic rendition of the HK/G6PD assay [11-13] of plasma diluted 109-fold in Tris/ HEPES-buffer ( $\mathrm{pH} 7.8 ; 83 / 5 \mathrm{mM})$ with $\mathrm{Mg}^{2+}(4 \mathrm{mM})$, ATP (>1.4 mM), $\mathrm{NADP}^{+}(>0.83 \mathrm{mM})$, hexokinase (yeast) $(>1.4 \mathrm{U} / \mathrm{mL})$ and glucose6-phosphate dehydrogenase (Escherichia coli) ( $>2.5 \mathrm{U} / \mathrm{mL}$ ), measuring spectrophotometric absorptions at $340 \mathrm{~nm}$ for NADPH formation and at $700 \mathrm{~nm}$ for blank subtraction [Gluco-quant Glucose/HK cobas ${ }^{\circledR}$ (ref: 11929534) version 2011-02, V 15]. The Roche C.f.a.s. calibrator (lot: 158689) with an assigned glucose value of $193 \mathrm{mg} / \mathrm{dL}$ (10.7 mM) is traceable to isotope dilution mass spectrometry (ID-MS) [expanded uncertainty $0.8 \%, \mathrm{k}=2$; Instruction sheet calibrator for automated

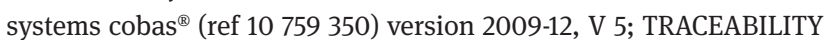
and UNCERTAINTY cobas c501/ c502 / c311 / c701 / c702 - C.f.a.s. Cat. No. 10759350 190, version 7 March 2011].

The comparison lactate assay on the Dimension RxL analyzer (Siemens Healthcare) is a modified Marbach and Weil end-point assay using lactate dehydrogenase [15] of plasma diluted 118-fold in Tris-buffer (0.24 mM, pH not specified) with dihydrazine sulphate (3.7 $\mu \mathrm{M})$, lactate dehydrogenase (rabbit muscle) $(19 \mathrm{U} / \mathrm{mL})$ and
$\mathrm{NAD}^{+}(2 \mu \mathrm{M})$, measuring spectrophotometric absorptions at $340 \mathrm{~nm}$ for NADH formation and at $383 \mathrm{~nm}$ for blank subtraction [Flex reagent cartridge, lactate, Dimension, Dade Behring (ref DF6), version 2003-09]. The three Siemens CHEM I CAL calibrators with assigned lactate values of 0.0, 8.2 and $16.6 \mathrm{mmol} / \mathrm{L}$ (no uncertainties assigned) (lot: 1CD038) are traceable to lithium lactate (source or purity not given; Siemens Dimension ${ }^{\circledR}$ clinical chemistry system CHEM I CAL (ref DC18A) version 2008-04).

All BGAs use glucose oxidase and lactate oxidase to quantify glucose and lactate, respectively.

\section{Linearity and recovery}

Heparinized venous blood was drawn from healthy adult volunteers. We added 22.5 g/L L-glyceraldehyde (G5001, Sigma-Aldrich, Zwijndrecht, The Netherlands) to stabilize glucose [16]. We confirmed that glyceraldehyde did not interfere with glucose and lactate assays. We prepared stock solutions of $2500 \mathrm{mg} / \mathrm{dL}$ of D-(+)-glucose (Sigma-Aldrich; product number: G8270, $\geq 99.5 \%$ purity) and of $170 \mathrm{mM} \mathrm{L-(+)-lactic} \mathrm{acid} \mathrm{(Sigma-}$ Aldrich; product number: L6402, $\geq 98 \%$ purity) in a homemade KrebsHenseleit buffer [17]. We added $210 \mu \mathrm{L}$ of isotonic mixtures of stock solution and Krebs-Henseleit buffer to $1500 \mu \mathrm{L}$ of a mixture of equal volumes of plasma and packed red blood cells (from the same donor) resulting in a hematocrit of $45 \%$. The concentration of analyte in total plasma was corrected $(+12.3 \%)$ for the volume occupied by hemoglobin inside erythrocytes [18]. We accounted for an extra-/intracellular distribution ratio (in the aqueous phase) of lactic acid of 1.59/1 assuming an extra- and intracellular $\mathrm{pH}$ and $\mathrm{a} \mathrm{pK}_{\mathrm{a}}$ value of lactic acid of 7.4, 7.2 and 3.86 , respectively [19] and an arbitrary $7 \%$ correction for equilibration with pyruvate assuming a lactate/pyruvate ratio of 13 [20].

Concentrations of the spikes are reported in Table 1. BGAs report in mass per total volume of plasma [21] comparable with the comparison methods, which measure the analyte in diluted plasma. Measurements were performed in duplicate.

\section{Imprecision}

Imprecision was evaluated from results for QC materials. Radiometer provided three levels (S7735 lot number: 433; S7745 lot number: 427; S7755 lot number: 310) and two levels (S9040 and S9050, lot number: 36738) for ABL800 and ABL90, respectively. Siemens provided three levels of QC material (lot number 136887).

Repeatability of the glucose and lactate assay was also evaluated by duplicate measurements on patient samples.

\section{Relative accuracy by method comparison}

During 1 week, 149 heparinized arterial blood samples for blood gas analysis, and sodium fluoride and potassium oxalate plasma samples were drawn by skilled nurses via an indwelling arterial line from 25 patients hospitalized in a tertiary referral surgical ICU at $12 \mathrm{am}$ and $4 \mathrm{pm}$. After discarding at least four times the internal volume of the arterial catheter and lining, a blood sample was drawn with a blood gas syringe (Pico syringe, Radiometer Medical ApS). Immediately after sampling (within 30 s), we analyzed arterial 
Table 1 Linearity and recovery experiments for glucose and lactate: basal concentrations in sample spiked with pure Krebs-Henseleit were for glucose 65 (3.61), 71 (3.94), 70 (3.89), 63 (3.50) mg/dL (mmol/L) and for lactate 1.96, 2.1, 1.8, $2.16 \mathrm{mmol} / \mathrm{L}$ on Modular P or Dimension RxL, ABL800, ABL90 and RP500, respectively. The polynomial regressions fit the recovered amount (replicates=2) vs. the added spike.

\begin{tabular}{|c|c|c|c|c|c|c|c|c|c|c|c|}
\hline \multirow{2}{*}{$\begin{array}{l}\text { Glucose } \\
\text { Spike, } \mathrm{mg} / \mathrm{dL} \\
(\mathrm{mmol} / \mathrm{L})\end{array}$} & \multicolumn{2}{|c|}{ Modular P } & \multicolumn{3}{|c|}{ ABL800 } & \multicolumn{3}{|r|}{ ABL90 } & \multicolumn{3}{|r|}{ RP500 } \\
\hline & Recovery, \% & CV, \% & \multicolumn{2}{|c|}{ Recovery, \% } & CV, & \multicolumn{2}{|c|}{ Recovery, \% } & \multicolumn{2}{|l|}{ CV, \% } & Recovery, \% & CV, \% \\
\hline $50(2.8)$ & 99.9 & 3.4 & & 104.0 & & & 105.0 & 0.0 & & 102.0 & 1.4 \\
\hline $99(5.5)$ & 95.5 & 1.3 & & 102.5 & & & 103.5 & 0.0 & & 98.0 & 2.2 \\
\hline 149 (8.3) & 97.7 & 0.7 & & 104.4 & & & 105.4 & 0.5 & & 103.4 & 1.4 \\
\hline $199(11.1)$ & 98.8 & 0.0 & & 104.0 & & & 105.5 & 1.0 & & 99.5 & 1.1 \\
\hline $249(13.8)$ & 97.4 & 0.4 & & 103.0 & & & 105.2 & 0.3 & & 94.8 & 2.7 \\
\hline $298(16.6)$ & 94.4 & 0.3 & & 100.8 & & & 102.3 & 0.2 & & 96.1 & 1.5 \\
\hline 348 (19.3) & 95.5 & 1.1 & & 103.0 & & & 103.9 & 0.4 & & 99.0 & 2.9 \\
\hline Average & 97.0 & 1.5 & & 103.1 & & & 104.4 & 0.5 & & 99.0 & 2.0 \\
\hline Best fit $(y=)$ & \multicolumn{3}{|c|}{$3.57+0.95 x$} & \multicolumn{3}{|c|}{$1.85+1.020 x$} & & $1.032 x$ & \multicolumn{3}{|c|}{$-17.39+1.47 x-0.0030 x^{2}+5.22^{\star} 10^{-6} x^{3}$} \\
\hline Lactate & \multicolumn{3}{|c|}{ Dimension RxL } & \multicolumn{3}{|c|}{ ABL800 } & & \multicolumn{2}{|c|}{ ABL90 } & \multicolumn{2}{|r|}{ RP500 } \\
\hline Spike, $\mathrm{mmol} / \mathrm{L}$ & Recovery, \% & & CV, \% & Recover & $\%$ & CV & Recovery, \% & & V, \% & Recovery, \% & CV, \% \\
\hline 3.64 & 102.1 & & 0.4 & & 3.8 & 2.3 & 87.9 & & 0.0 & 98.5 & 2.0 \\
\hline 7.27 & 99.4 & & 0.4 & & 3.2 & 3.5 & 85.3 & & 2.3 & 84.5 & 4.8 \\
\hline 10.91 & 96.5 & & 0.4 & & 7.0 & 0.0 & 78.8 & & 0.0 & 86.5 & 4.9 \\
\hline 14.55 & 94.7 & & 0.7 & & 8.7 & 0.6 & 75.6 & & 0.0 & 79.8 & 3.5 \\
\hline 18.18 & 92.2 & & 0.5 & & 9.2 & 4.9 & 75.4 & & 5.2 & 80.6 & 0.9 \\
\hline 21.82 & 90.2 & & 1.1 & & 9.7 & 4.1 & 74.2 & & 0.0 & 80.4 & 7.9 \\
\hline 25.45 & 94.3 & & 0.9 & & 8.2 & 0.0 & 71.5 & & 0.0 & 74.8 & 6.4 \\
\hline Average & 95.6 & & 0.7 & & 0.0 & 2.9 & 78.4 & & 2.1 & 83.6 & 4.9 \\
\hline Best fit $(y=)$ & \multicolumn{3}{|c|}{$-0.85+1.36 x-0.039 x^{2}+0.00093 x^{3}$} & \multicolumn{3}{|c|}{$0.20+0.78 x$} & \multicolumn{3}{|c|}{$0.44+0.79 x-0.0035 x^{2}$} & & $1.09+0.73 x$ \\
\hline
\end{tabular}

blood glucose and lactate sample by sample on ABL800, ABL90 or RP500. The analyses were repeated on all BGAs in a second round to assess repeatability of glucose and lactate measurements in blood of ICU patients. As the time to ready differs for these analyzers, and the order was dictated by their readiness the scheme resulted in a pseudo-randomized order, which cancelled out systematic effects of any possible glucose consumption on the variance and/or relative bias between analyzers. For method comparison purposes the first of the duplicate measurements was used in the calculations, avoiding any unfair effects of averaging with comparison to real-life use of the equipment. The results of the duplicate measurements turned out to be equivalent (see Results section).

Standard tubes for quantification of plasma glucose (sodium fluoride and potassium oxalate [22]) were sampled immediately after the arterial blood gases and within $<3$ min were centrifuged at $3200 \mathrm{rpm}$ for $10 \mathrm{~min}$ at $4^{\circ} \mathrm{C}$ to maximally prevent glucose metabolism in the sample [23]. Supernatants were transferred to a fresh vial and sent to the central clinical laboratory via a pneumatic transport system to be analyzed with the comparison methods within $20 \mathrm{~min}$.

\section{Statistical methods}

Calculations were performed in Microsoft Excel 2010 [version 14.0.6112.5000 (32-bits)] with use of the Analyse-it (version 2.26 Excel10+) add-on where indicated. For maximum acuity of derived statistics the results were collected with the maximum of positions after the decimal point that each BGA allowed (glucose: 0 for ABL800, ABL90, RP500 and 1 for Modular P; lactate: 1 for ABL800, ABL90 and 2 for RP500, Dimension RxL). Where the rounding was too liberal, the resulting figures show a layered distribution of data.

Linearity was evaluated according the CLSI guideline EP6-A [24] by polynomial regression using Analyse-it. Recovery expressed as the fractional difference between the average result of replicate measurements and the nominal value of the spiked as well as the coefficient of variation (CV\%, from the replicate measurements) are reported in Table 1. The minimum order to explain the data was determined and reported as the best fit for glucose and lactate on the various analyzers, respectively (Table 1).

Repeatability, estimated from replicate analysis of QC materials and patient samples (or 'within-run') $\left(\mathrm{CV} \%{ }_{\mathrm{r}}\right)$, within-day $\left(\mathrm{CV} \%{ }_{\text {wd }}\right)$ and between-day $\left(\mathrm{CV} \% \%_{\text {bd }}\right)$ imprecision, as well as total imprecision $\left(\mathrm{CV} \%_{\text {total }}\right)$ were calculated according to the CLSI guideline EP5-A2 [25] (Tables 2 and 3). Moreover, for the $\mathrm{CV} \%{ }_{\mathrm{r}}$ on patient samples a second degree polynomial was fitted to the individual $\mathrm{CV} \% \mathrm{~s}$ as a function of the averaged level measured (Figure 1).

Differences between duplicate results were appreciated at a twosided significance level of 0.05 via paired t-test.

The correlation of results between methods was analyzed by orthogonal linear regression (Figures 2 and 3, panels A-C; null hypothesis: solid line, the fitted regression line: dashed line). The calculus was validated using data-sets from Geigy Scientific Tables [26]. The 95\% confidence interval on the estimates of slope and intercept and the joint 95\% confidence ellipse are shown in a X-Y plot (Figures 2 and 3, Panels D-F) [27]. The calculus was validated using published data-sets [28]. 
A

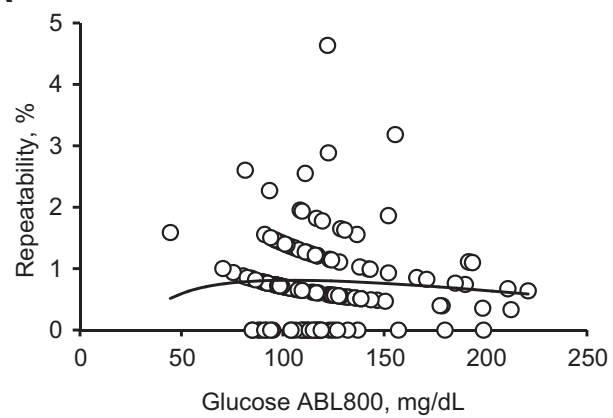

B

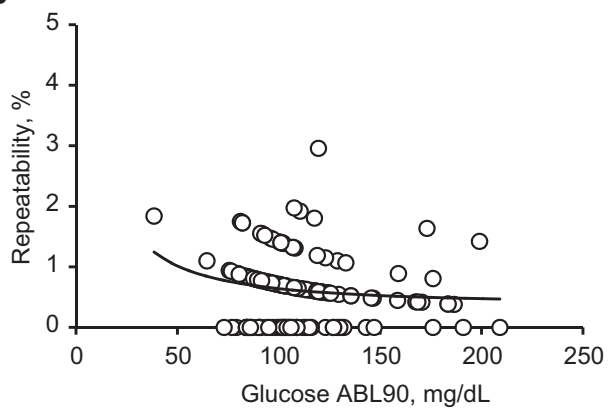

C

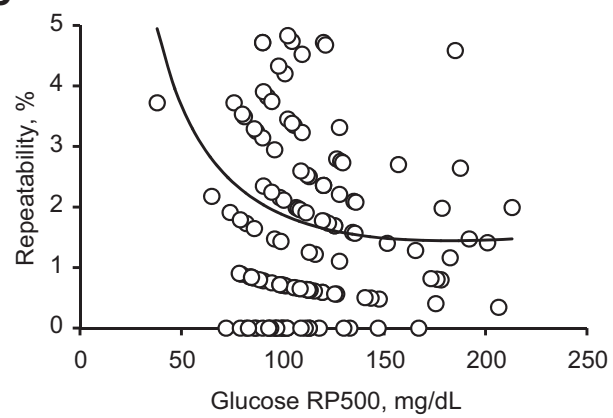

D

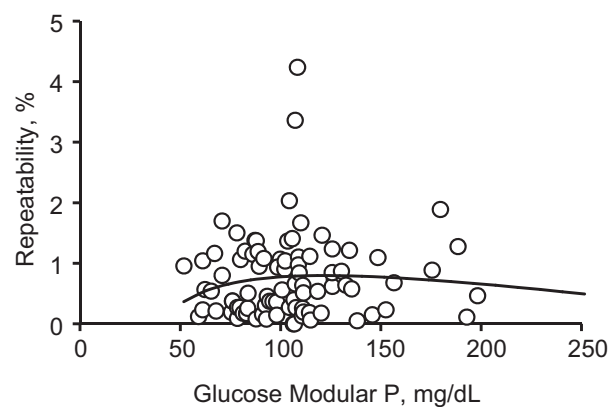

E

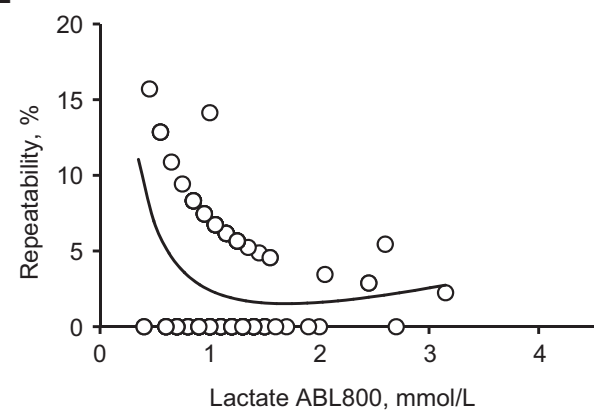

F

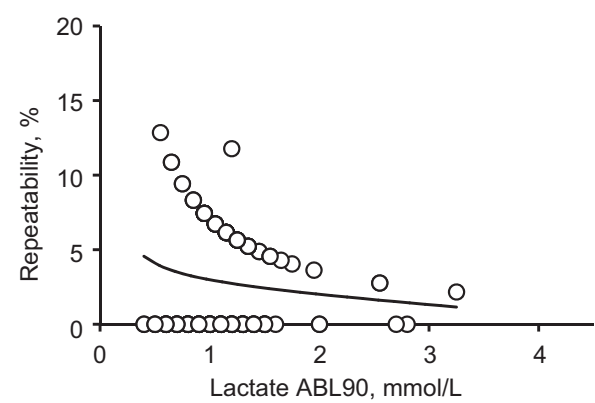

G

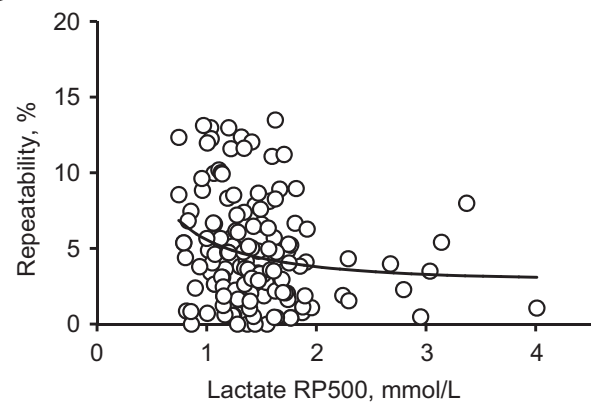

H

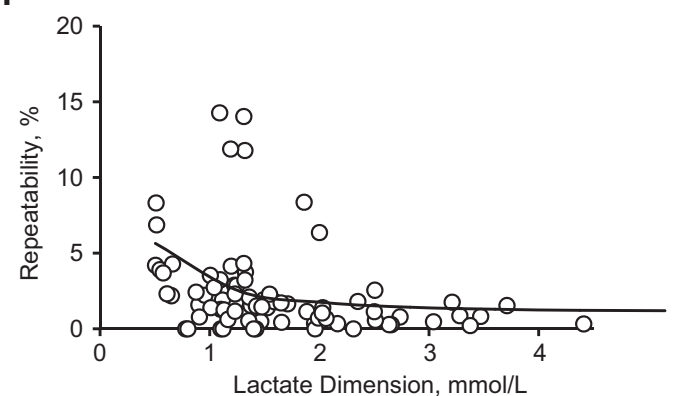

Figure 1 Repeatability for glucose and lactate.

Repeatability for glucose (A-D) and lactate $(E-H)$ for BGAs and the comparison platforms is shown. The estimated coefficient of variation is plotted as a function of their average value. For the BGAs samples stem from the method comparison studies. For the comparison platforms an equivalent number of routine samples was used. A second degree polynomial fit through the data is shown (solid line). Excessive rounding of results, removing significant digits, results in layered patterns.

For the evaluation of bias with respect to the comparison method we constructed a Bland-Altman-like residual plot (Figures 2 and 3, Panel $\mathrm{G}-\mathrm{I}$ ) of the orthogonal distances (error) of data points to the fit [29]. Bias was evaluated by comparison with the null-hypothesis (as solid line: with intercept $=0$, and slope $=1$ ) with indication of the total allowable error interval. The criteria for and the construction of the error interval are discussed in the results and discussion sections. Whether the number of samples falling inside or outside the predefined total error limits was significantly different for the platforms tested was analyzed in a $2 \times 3$ contingency table by Pearson's $\chi^{2}$ analysis in Analyse-it. 
A

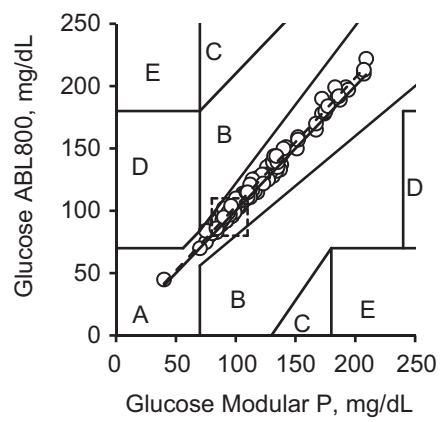

B
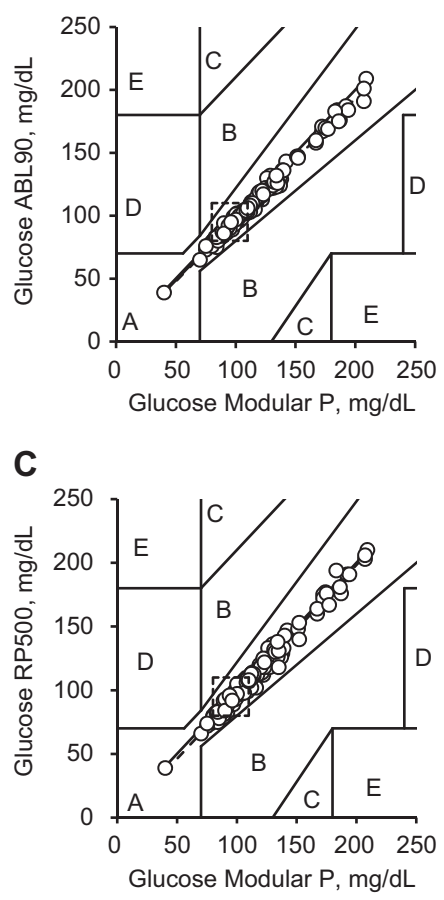

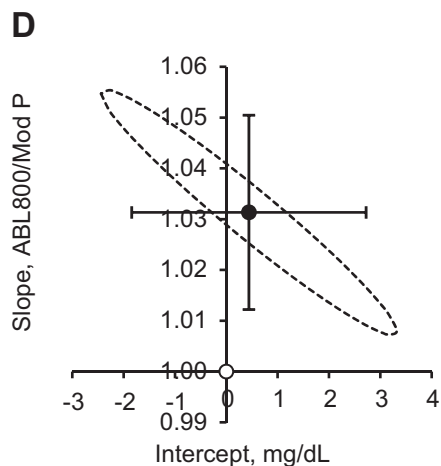

E
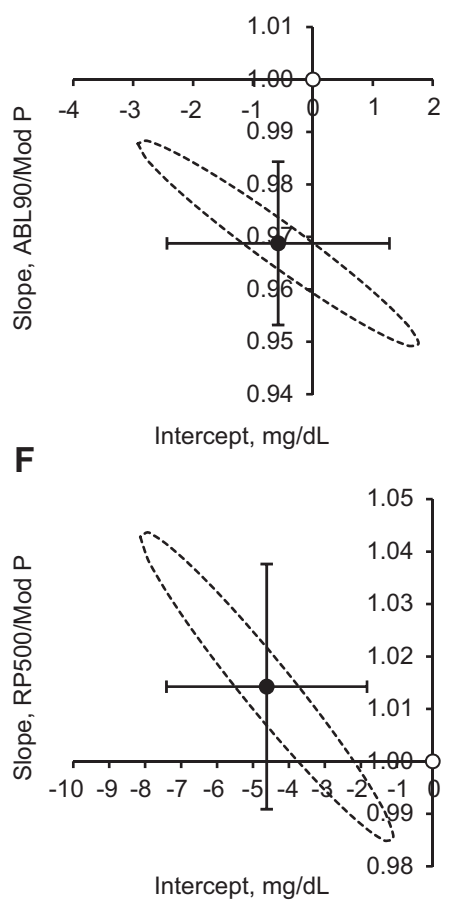

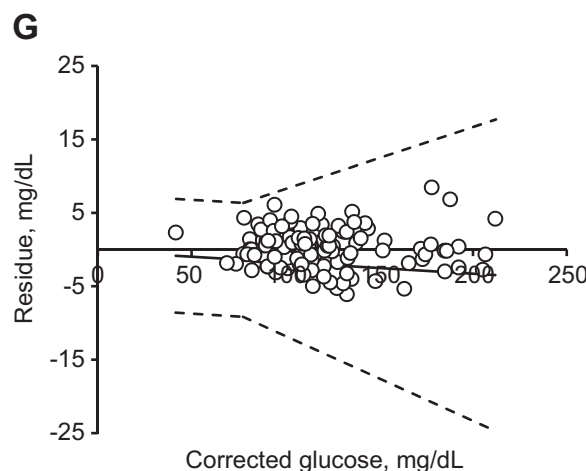

H

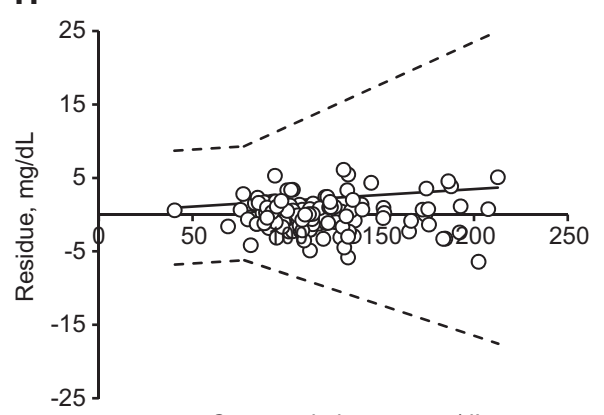

Corrected glucose, $\mathrm{mg} / \mathrm{dL}$

I

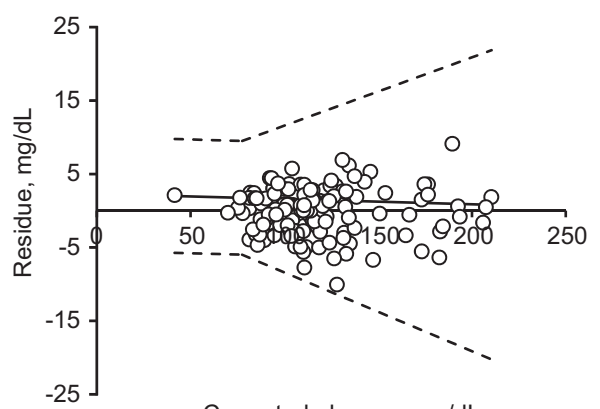

Corrected glucose, $\mathrm{mg} / \mathrm{dL}$

Figure 2 Method comparisons for glucose.

From top to bottom we compare ABL800, ABL90 and RP500 with Modular P. (A-C): null hypothesis (solid line) and regression analysis (dashes) with the tight glucose control target range (dashed square) and the Clarke error grid; (D-F): null hypothesis (O) and the intercept and slope () with $95 \%$ joint confidence interval (ellipse); (G-I): residual plot: orthogonal distance (y-axis) of data points to their projection $(x$-axis) on the regression line. The tolerable error interval is given in dashed lines.

\section{Results}

\section{Performance of glucose measurement}

\section{Linearity and recovery}

Polynomial regression revealed a linear relationship (first order model) for glucose measurement on Radiometer BGAs and the comparison method (Table 1). A third order polynomial was needed for RP500. Recoveries were within $5 \%$ of the expected value.

\section{Imprecision}

Imprecision from QC measurements was comparable for all BGAs and the comparison method (Table 2). Mean repeatability determined on patient samples was comparable among ABL800 and ABL90 (CV\% $\%_{\mathrm{r}-\mathrm{ABL} 800}=1.1 \%$; CV\% $\%_{\mathrm{r}-\mathrm{ABL} 90}$ $=0.8 \%)$ and the comparison method $\left(\mathrm{CV} \%{ }_{\mathrm{r} \text {-Modularp }}\right.$ $=1.0 \%)$, but higher for RP500 $\left(\mathrm{CV} \%_{\mathrm{r} \cdot \mathrm{RP} 500}=2.4 \%\right)$ (Figure 1A-D).

Glucose did not decline in between duplicate analyses of patient samples on ABL800 [mean glucose levels \pm standard deviation: $118.95 \pm 30.51 \mathrm{mg} / \mathrm{dL}(6.61 \pm 1.70 \mathrm{mmol} / \mathrm{L})$ 
Table 2 Bias and imprecision data for glucose measurements.

\begin{tabular}{|c|c|c|c|c|c|c|c|}
\hline \multirow[b]{2}{*}{ Glucose } & \multirow[b]{2}{*}{$\begin{array}{r}\text { Sample mean (SD) } \\
\mathrm{mg} / \mathrm{dL}(\mathrm{mmol} / \mathrm{L})\end{array}$} & \multicolumn{2}{|c|}{ Patient samples mean bias estimates } & \multicolumn{4}{|c|}{ Quality control materials imprecision estimates } \\
\hline & & Range mg/dL (mmol/L) & $\begin{array}{r}\text { Mean difference } \\
\text { with Modular (\%) }\end{array}$ & $\begin{array}{r}\mathrm{CV} \%_{\text {total }}^{\mathrm{b}}(\mathrm{TV}) \mathrm{mg} / \mathrm{dL} \\
(\mathrm{mmol} / \mathrm{L})\end{array}$ & $\mathrm{CV} \%_{\mathrm{r}}{ }^{\mathrm{d}}$ & $C V \%_{w d}^{e}$ & $\mathrm{CV}_{\text {bd }}{ }^{\mathrm{f}}$ \\
\hline Modular P & $115(30)[6.39(3.33)]$ & $39-209(2.11-11.61)$ & - & - & - & - & - \\
\hline \multirow[t]{3}{*}{$\mathrm{ABL} 800$} & $120(31)[6.67(1.72)]$ & & $+4.3^{\mathrm{g}}$ & $2.2[28(1.56)]$ & 1.4 & 1.8 & 0.0 \\
\hline & & & & $1.6[101(5.61)]$ & 0.9 & 1.3 & 0.0 \\
\hline & & & & $1.2[241(13.39)]$ & 0.7 & 1.0 & 0.0 \\
\hline \multirow[t]{2}{*}{ ABL90 } & $111(29)[6.12(1.61)]$ & & $-3.5^{\mathrm{g}}$ & $0.8[130(7.22)]$ & 0.3 & 0.4 & 0.6 \\
\hline & & & & $0.9[269(14.94)]$ & 0.3 & 0.6 & 0.5 \\
\hline \multirow[t]{3}{*}{ RP500 } & $113(31)[6.28(1.72)]$ & & $-1.7^{\mathrm{g}}$ & $1.6[52(2.89)]$ & 0.7 & 0.9 & 1.1 \\
\hline & & & & $0.6[103(5.72)]$ & 0.2 & 0.4 & 0.3 \\
\hline & & & & $0.3[206(11.44)]$ & 0.3 & 0.1 & 0.2 \\
\hline
\end{tabular}

a Standard deviation; ${ }^{\mathrm{b} C o e f f i c i e n t}$ of variance; ' Target value; ${ }^{\mathrm{d}}$ Repeatability; ${ }^{\mathrm{e} W i t h i n-d a y ; ~}{ }^{\mathrm{f} B e t w e e n}$-day. ${ }^{\mathrm{g}} \mathrm{p}<0.05$ (paired t-test).

and $118.99 \pm 30.82 \mathrm{mg} / \mathrm{dL}(6.61 \pm 1.71 \mathrm{mmol} / \mathrm{L})$ for the first and second round, respectively], ABL90 [110.32 \pm 28.30 $\mathrm{mg} / \mathrm{dL}(6.13 \pm 1.57 \mathrm{mmol} / \mathrm{L})$ and $109.82 \pm 28.34 \mathrm{mg} / \mathrm{dL}$ $(6.10 \pm 1.57 \mathrm{mmol} / \mathrm{L})]$ or $\mathrm{RP} 500 \quad[111.87 \pm 29.89 \mathrm{mg} / \mathrm{dL}$ $(6.22 \pm 1.66 \mathrm{mmol} / \mathrm{L})$ and $111.50 \pm 30.40 \mathrm{mg} / \mathrm{dL}(6.19 \pm 1.69$ $\mathrm{mmol} / \mathrm{L})]$. None of these differences became significant in a paired t-test analysis $(\mathrm{p}<0.05)$. Thus, glucose levels remained stable within the timeframe of our study $(7 \mathrm{~min}$ per sample). This validated our method comparison studies (see next section).

\section{Method comparison}

Orthogonal regression analysis revealed a good agreement of glucose measurements for all BGAs with the comparison method (Figure 2A-C). In the Clarke error grid analysis, all results were situated in zone A excluding relevant error in routine clinical applications. For all BGAs, intercept and slope of the estimated regression lines differed significantly from the null-hypothesis (intercept $=0 \mathrm{mg} / \mathrm{dL}$, and slope $=1$; Figure 2D-F). The population average for both the method under study (y-axis) and the comparison method (x-axis) was used to calculate an average fractional bias between both methods (Table 2).

The clinical relevance of the observed analytical differences was assessed in a Bland-Altman-like plot (Figure 2G-I). The orthogonal distance of the observed data points to the fitted regression lines were plotted as a function of their projection on that regression line. The line of identity with the comparison method was plotted with an envelope of tolerable error around that line. This envelope was determined by requirements for correct insulin dosage during TGC [30]. Karon et al. specified that an error for glucose measurement below 10\% correlates with a frequency of major insulin dose errors of $<0.2 \%$ [30]. The envelope is constructed as follows. The center of the target range for TGC in ICU patients [1], $77.5 \mathrm{mg} / \mathrm{dL}$ $(4.31 \mathrm{mmol} / \mathrm{L})$, was used as a cut-off. Below the cut-off we allowed a constant error of $10 \%$ of that value $[7.75 \mathrm{mg} / \mathrm{dL}$ (0.43 mmol/L)], and above that cut-off a proportional error of $10 \%$. All observed residues were situated within the envelope for the ABL800, ABL90 and RP500 systems (Figure 2 right hand column).

\section{Performance of lactate measurement}

\section{Linearity and recovery}

A linear regression explained the relationship between lactate spiked and recovered on the ABL800 and RP500 BGAs (Table 1). Higher order polynomials were needed for ABL90 and the comparison method (Table 1). For all BGAs and the comparison method, recovery of lactate decreased as the amount of lactate added increased (Table 1).

\section{Imprecision}

Imprecision evaluated from QC results were comparable for all BGAs and the comparison method (Table 3). Mean within-run imprecision, from duplicate analysis of patient samples, was comparable among ABL800 and ABL90 $\left(C V \%_{\mathrm{r}-\mathrm{ABL} 800}=4.0 \%\right.$; CV\% $\left.\%_{\mathrm{r}-\mathrm{ABL} 90}=4.2 \%\right)$ and the comparison method $\left(\mathrm{CV} \%_{\mathrm{r}-\text { Dimension }}=3.5 \%\right)$ but higher for RP500 $\left(\mathrm{CV} \%_{\mathrm{r}-\mathrm{RP} 500}=5.6 \%\right)($ Figure $1 \mathrm{E}-\mathrm{H})$. These CV\%s were contaminated with a systematic (albeit smaller than the here reported CV\%s) rise in lactate levels. Indeed, paired t-test analysis of first and second duplicate results of patient 
Table 3 Bias and imprecision data for lactate measurements.

\begin{tabular}{|c|c|c|c|c|c|c|c|}
\hline \multirow[b]{2}{*}{ Lactate } & \multirow[b]{2}{*}{$\begin{array}{l}\text { Sample mean } \\
\left(S^{a}\right) \mathrm{mmol} / \mathrm{L}\end{array}$} & \multicolumn{2}{|c|}{ Patient samples mean bias estimates } & \multicolumn{4}{|c|}{ Quality control materials imprecision estimates } \\
\hline & & $\begin{array}{l}\text { Range, } \\
\mathrm{mmol} / \mathrm{L}\end{array}$ & $\begin{array}{r}\text { Mean difference } \\
\text { with Dimension, \% }\end{array}$ & $\begin{array}{r}\mathrm{CV} \%^{\mathrm{b}}{ }_{\text {total }}(\mathrm{TV}) \\
\mathrm{mmol} / \mathrm{L}\end{array}$ & $\mathrm{CV} \%_{r}{ }^{d}$ & $C V \%_{\text {wd }}{ }^{e}$ & $\mathbf{C V} \%{ }_{\text {bd }}{ }^{\mathrm{f}}$ \\
\hline Dimension & $1.21(0.49)$ & $0.35-3.81$ & - & - & - & - & - \\
\hline \multirow[t]{3}{*}{ ABL800 } & $1.12(0.42)$ & & $-7.4^{\mathrm{g}}$ & $3.3(1.6)$ & 1.9 & 2.7 & 0.0 \\
\hline & & & & $2.1(4.5)$ & 1.1 & 1.9 & 0.0 \\
\hline & & & & $2.4(10.5)$ & 0.9 & 2.2 & 0.0 \\
\hline \multirow[t]{2}{*}{ ABL90 } & $1.11(0.49)$ & & $-8.2^{\mathrm{g}}$ & $2.7(3.2)$ & 0.9 & 1.0 & 2.4 \\
\hline & & & & $1.9(7.0)$ & 0.6 & 0.5 & 1.7 \\
\hline \multirow[t]{3}{*}{ RP500 } & $1.43(0.50)$ & & $+18.2^{\mathrm{g}}$ & $4.9(0.9)$ & 1.7 & 4.1 & 2.2 \\
\hline & & & & $4.5(3.1)$ & 1.5 & 1.8 & 3.8 \\
\hline & & & & $3.4(12.8)$ & 2.2 & 1.8 & 1.9 \\
\hline
\end{tabular}

${ }^{\mathrm{a}}$ Standard deviation; ${ }^{\mathrm{b} C o e f f i c i e n t}$ of variance; ${ }^{\mathrm{T}}$ Target value; ${ }^{\mathrm{d}}$ Repeatability; ${ }^{\mathrm{e}}$ Within-day; ${ }^{\mathrm{f}}$ Between-day. ${ }^{\mathrm{g}} \mathrm{p}<0.05$ (paired t-test).

samples became significant. The first and second results, respectively, were $1.12 \pm 0.42$ (mean \pm standard deviation) vs. $1.14 \pm 0.43 \mathrm{mmol} / \mathrm{L}, 1.11 \pm 0.44$ vs. $1.15 \pm 0.44 \mathrm{mmol} / \mathrm{L}$, $1.43 \pm 0.50$ vs. and $1.47 \pm 0.49 \mathrm{mmol} / \mathrm{L}$ on ABL800, ABL90 and RP500, respectively. The rise could be detected before a relevant decrease in glucose levels was observed, notwithstanding a less favorable CV\% for the analysis of lactate. This might be explained by the fact that 1 mole of glucose yields up to 2 moles of lactate (if produced entirely by lactic fermentation in obligatory anaerobic erythrocytes) and that this increment was seen with respect to low off-set values. Lactate levels did not rise in between duplicate analyses of patient samples on the comparison method ( $1.17 \pm 0.43$ vs. $1.15 \pm 0.45 \mathrm{mmol} / \mathrm{L})$, as plasma had been separated from the metabolically active blood cell components.

\section{Method comparison}

Orthogonal regression analysis (Figure $3 \mathrm{~A}-\mathrm{C}$ ) revealed an acceptable correlation for lactate measurements between the ABL800 and ABL90 BGA and the comparison method $\left(r^{2}>0.93\right)$, while the lactate measurement on RP500 correlated less well with the comparison method $\left(\mathrm{r}^{2}=0.85\right)$.

Intercept and slope of the estimated regression lines differed significantly from the null-hypothesis (intercept $=0 \mathrm{mg} / \mathrm{dL}$, and slope $=1$; Figure 3D-F). Mean values for lactate measurement on patient samples with ABL800, ABL90 and RP500 were 7.4\%, 8.2\% lower and 18.2\% higher than the comparison measurement on the Dimension RxL, respectively (Table 3).

In the Bland-Altman-type plot (Figure 3G-I) we projected observed data points vis-à-vis the tolerable error. The tolerated deviance with respect to the null-hypothesis (line of identity with the comparison method) was constructed as follows: below $1.2 \mathrm{mM}$ [center of reference range; Flex reagent cartridge, lactate, Dimension, Dade Behring (ref. DF6), version 2003-09] we allowed a constant error of $30 \%$ (desirable total error) [31] of that value $(0.36 \mathrm{mM})$, and above the $1.2 \mathrm{mM}$ cut-off a proportional error of $30 \%$. One hundred percent, $100 \%$, and $98.7 \%$ of the observed residues were situated within the predetermined limits for ABL800, ABL90 and RP500, respectively. Among those BGAs, no difference was observed in the proportion of data lying outside the predetermined limits ( $p>0.05)$.

\section{Discussion}

\section{Glucose measurements}

Our repeatability was for all BGAs $<2.5 \%$, and better than what we reported for ABL700 [32]. Others [33] reported a comparable imprecision profile (CV\%) for the Siemens RP405 cassette-based system, but inferior precision for ABL90. In our hands the ABL90 and RP500 showed a bias relative to the ABL800 of $1.3 \%$ and $-4.1 \%$, respectively. Others [33] reported a bias for ABL90 and RP405 relative to ABL700 of $-4.43 \%$ and $+0.31 \%$, respectively.

The evaluation of fitness for purpose requires the definition of quality specifications. Ricos et al. defined that desirable repeatability and total allowable error should be below $2.9 \%$ and $6.9 \%$, respectively, based on intra- and inter-individual biological variation in a healthy population [31]. The Royal College of Pathologists of Australasia (RCPA) proposes criteria that reach outside the normal physiological range [34]. They define a cut-off value below which a constant - and above which a proportional error 

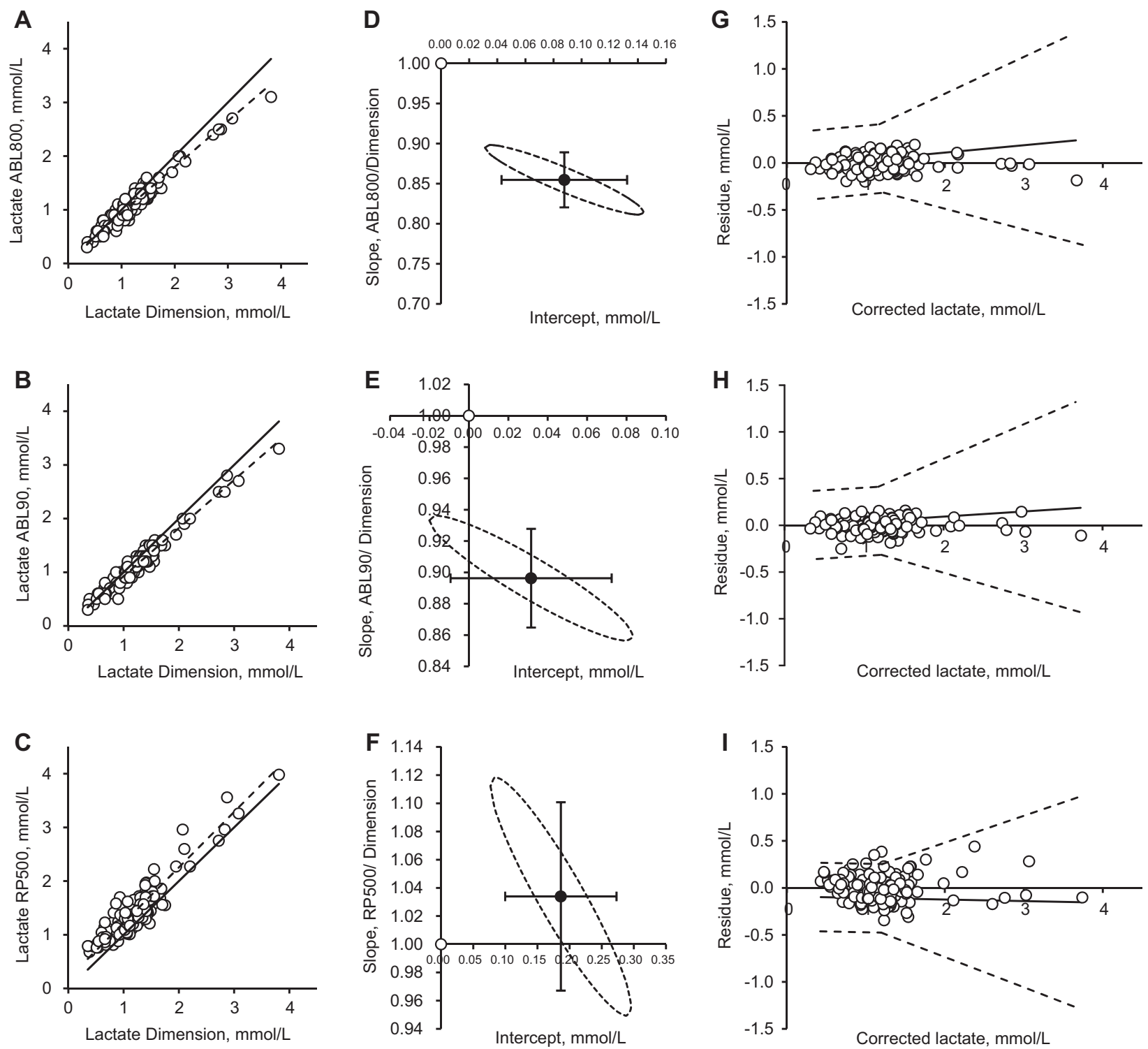

Figure 3 Method comparisons for lactate.

From top to bottom we compare ABL800, ABL90 and RP500 with Dimension RxL. (A-C): null hypothesis (solid line) and regression analysis (dashes); (D-F): null hypothesis (O) and the intercept and slope (O) with $95 \%$ joint confidence interval (ellipse); (G-I): residual plot: orthogonal distance (y-axis) of data points to their projection (x-axis) on the regression line. The tolerable error interval is given in dashed lines.

is tolerated (see Figures 2 and 3). The resulting trumpetlike error profile coincides shape-wise with profiles for clinical error as obtained by consensus voting [34]. In the current study we chose for glucose an allowable error of $10 \%$, based on a simulation study of Karon et al. [30]. These authors evaluated the proportion of serious insulin dosing errors in a simulated TGC protocol. The proposed criteria reduced serious insulin dosing errors to $<0.2 \%$ [30]. The $10 \%$ error allowed in the current paper would lead to a rate of hypoglycemia between $0 \%$ and $0.2 \%$ and a rate of hyperglycemia between $0 \%$ and $10 \%$, based on simulation of the Yale insulin regimen in an ICU [10]. Given this criterion, we could then calculate the fraction of results outside the pre-set quality requirement boundaries. As for all BGAs all measurements fell within the tolerable error boundaries, we concluded that cassette-based as well as traditional BGAs are able to accurately quantify blood glucose concentrations in an ICU setting for the purpose of TGC.

\section{Blood gas analyzers vs. handheld glucose meters}

Handheld glucose meters perform worse in a setting of TGC, with bias as high as $15 \%-32 \%[8,35,36]$ and, based 
on within- and among-strip variability, a total imprecision of $5 \%-11 \%$ [8]. Based on simulation modeling, use of glucose meters might degrade TGC in the ICU [10]. On the pre-analytical site, finger-stick samples are unreliable in critically ill patients, as difference in skin temperature or local perfusion induces bias for glucose vs. the central laboratory analyzers [37-39]. Accordingly, FDA advices that portable glucose meters are unsuitable in critically ill patients [39].

\section{Lactate measurements}

For ABL90 CV\% from $1.6 \%$ to $2.4 \%$ were determined on QC material [33]. Recent studies reported biases of $-12 \%$ and $-25 \%$ for ABL700 and ABL90, respectively, vs. a lactate oxidase assay-based comparison method [33, 40]. Lactate measurement was only recently (mid 2011) introduced on the RP500 cassette-based BGA platform. To our knowledge, the present study is the first to compare the lactate measurement on RP500 with a comparison method.

In the recovery experiment (Table 1) the RP500 seemed to outperform the ABL systems, while the opposite was true in the method comparison (Figure 3) and imprecision studies (Table 3). In the recovery experiments the actual value of plasma lactate is uncertain due to the actual distribution of the lactate in whole blood over cellular and metabolic compartments. The algebraic order of the bias was identical in the recovery experiment and the method comparison experiment: in each experiment the RP500 produced on average the higher values. The traceability of the lactate calibrators is uncertain (see Materials and methods section).

To evaluate fitness for purpose we based our criterion $(30 \%)$ on the desirable total error concept of Ricos et al. [31] as studies comparable to the glucose-studies of Boyd [10] and Karon [30] are lacking. The cut-off value where the precision profile changes character is set to the middle of the reference range for controls. Given this acceptance criterion, the ABL800 and ABL90 tended to outperform the RP500 system (Figure 3). Besides a lower constant bias in this experiment, the lower scatter also appeared to be an important factor contributing to an overall good performance.

\section{Conclusions}

The measurement of glucose and lactate with cassettebased BGAs is clinically acceptable and the results are comparable with the comparison method. As analytical performance is one of the variables determining clinical outcome, the use of ABL90 and RP500 cassette-based BGA for glucose and lactate measurement might highly reduce the impact of the analytical variability on clinical outcome.

\section{Conflict of interest statement}

Authors' conflict of interest disclosure: The authors stated that there are no conflicts of interest regarding the publication of this article. Research funding played no role in the study design; in the collection, analysis, and interpretation of data; in the writing of the report; or in the decision to submit the report for publication.

Research funding: The manufacturers provided BGAs and consumables free of charge (Radiometer Benelux, Zoetermeer, The Netherlands; Siemens Healthcare Diagnostics, Anderlecht, Belgium). All experiments and the preparation of this report were conducted without interference by the manufacturers.

Employment or leadership: K.P. received travel grants from Radiometer and Siemens companies. Other authors declare to have no conflicts of interest.

Honorarium: None declared.

Received December 4, 2012; accepted February 4, 2013; previously published online March 14, 2013

\section{References}

1. Van den Berghe G, Wouters P, Weekers F, Verwaest C, Bruyninckx F, Schetz $M$, et al. Intensive insulin therapy in critically ill patients. $N$ Engl J Med 2001;345:1359-67.

2. Vlasselaers D, Milants I, Desmet L, Wouters PJ, Vanhorebeek I, van den Heuvel I, et al. Intensive insulin therapy for patients in paediatric intensive care: a prospective, randomised controlled study. Lancet 2009;373:547-56.
3. Van den Berghe G, Schetz M, Vlasselaers D, Hermans G, Wilmer A, Bouillon R, et al. Clinical review: intensive insulin therapy in critically ill patients: NICE-SUGAR or Leuven blood glucose target? J Clin Endocrinol Metab 2009;94:3163-70.

4. Agus MS, Steil GM, Wypij D, Costello JM, Laussen PC, Langer M, et al. Tight glycemic control versus standard care after pediatric cardiac surgery. N Engl J Med 2012;367:1208-19. 
5. Hirsch IB. Understanding low sugar from NICE-SUGAR. N Engl J Med 2012;367:1150-2.

6. Wiener RS, Wiener DC, Larson RJ. Benefits and risks of tight glucose control in critically ill adults: a meta-analysis. JAMA 2008;300:933-44.

7. Scott MG, Bruns DE, Boyd JC, Sacks DB. Tight glucose control in the intensive care unit: are glucose meters up to the task? Clin Chem 2009;55:18-20.

8. Kimberly MM, Vesper HW, Caudill SP, Ethridge SF, Archibold E, Porter KH, et al. Variability among five over-the-counter blood glucose monitors. Clin Chim Acta 2006;364:292-7.

9. Boyd JC, Bruns DE. Quality specifications for glucose meters: assessment by simulation modeling of errors in insulin dose. Clin Chem 2001;47:209-14.

10. Boyd JC, Bruns DE. Monte Carlo simulation in establishing analytical quality requirements for clinical laboratory tests: meeting clinical needs. Methods Enzymol 2009;467:411-33.

11. Neese JW, Duncan P, Bayse D, Robinson M, Cooper T, Stewart C. Development and evaluation of a hexokinase/glucose-6phosphate dehydrogenase procedure for use as a national glucose comparison method. HEW Publication No. (CDC) 77-8330. Atlanta: Centers for Disease Control and Prevention, 1976.

12. Kunst A, Draeger B, Ziegenhorn J. UV-methods with hexokinase and glucose-6-phosphate dehydrogenase. In: Bergmeyer HU, Bergmeyer J, Grassl M, editors. Methods of enzymatic analysis: Volume VI: Metabolites 1: Carbohydrates. Weinheim-Deerfield Beach-Basel: Verlag Chemie, 1981:164.

13. Pelletier O, Arratoon C. Precision of glucose measurements in control sera by isotope dilution/mass spectrometry: proposed definitive method compared with a comparison method. Clin Chem 1987;33:1397-402.

14. Noll F. L-(+)-lactate. In: Bergmeyer HU, Bergmeyer J, Grassl M, editors. Methods of enzymatic analysis: Volume VI: Metabolites 1: Carbohydrates. Weinheim-Deerfield Beach-Basel: Verlag Chemie, 1981:583.

15. Marbach EP, Weil MH. Rapid enzymatic measurement of blood lactate and pyruvate. Use and significance of metaphosphoric acid as a common precipitant. Clin Chem 1967;13:314-25.

16. Landt M. Glyceraldehyde preserves glucose concentrations in whole blood specimens. Clin Chem 2000;46:1144-9.

17. Krebs HA, Henseleit K. Untersuchungen über die Harnstoffbildung im Tierkörper. Hoppe-Seyler's Zeitschrift für Physiol Chemie 1932;210:33-66.

18. Wahl HG. How accurately do we measure blood glucose levels in intensive care unit (ICU) patients? Best Pract Res Clin Anaesthesiol 2009;23:387-400.

19. Wahl P, Zinner C, Zengyuan Y, Bloch W, Mester J. Warming-up affects performance and lactate distribution between plasma and red blood cells. J Sports Sci Med 2010;9:499-507.

20. De Pinieux G, Chariot P, Ammi-Saïd M, Louarn F, Lejonc JL, Astier A, et al. Lipid-lowering drugs and mitochondrial function: effects of HMG-CoA reductase inhibitors on serum ubiquinone and blood lactate/pyruvate ratio. Br J Clin Pharmacol 1996;42:333-7.

21. D’Orazio P, Burnett RW, Fogh-Andersen N, Jacobs E, Kuwa K, Külpmann WR, et al. Approved IFCC recommendation on reporting results for blood glucose. International Federation of Clinical Chemistry and Laboratory Medicine Scientific Division.
Working Group on Selective Electrodes and Point-of-Care Testing Approved IFCC recommendation on reporting results for blood glucose: International Federation of Clinical Chemistry and Laboratory Medicine Scientific Division, Working Group on Selective Electrodes and Point-of-Care Testing (IFCC-SD-WGSEPOCT). Clin Chem Lab Med 2006;44:1486-90.

22. Swaminathan R, Cockram CS. Effectiveness of sodium fluoride as a preservative of glucose in blood. Clin Chem 1989;35:315-7.

23. Seeley ES, Bowen R, Faix JD. Rapid blood separation is superior to fluoride for preventing in vitro reductions in measured blood glucose concentration. J Clin Pathol 2009;62:752-3.

24. NCCLS. Evaluation of the linearity of quantitative measurement procedure: a statistical approach; approved guideline. NCCLS document EP6-A. Wayne, PA: NCCLS, 2003.

25. NCCLS. Evaluation of the precision performance of quantitative measurement methods: approved guideline, 2nd ed. NCCLS document EP5-A2. Wayne, PA: NCCLS, 2004.

26. Lentner C, editor. Scientific tables, 8th ed. Basel: Geigy-Ciba, 1981.

27. Davis RB, Thompson JE, Pardue HL. Characteristics of statistical parameters used to interpret least-squares results. Clin Chem 1978;24:611-20.

28. Draper N, Smith H. Applied regression analysis, 2nd ed. New York: John Wiley and sons, 1981.

29. Deming WE. Statistical adjustment of data. New York: John Wiley and sons, 1943. (republished in 1985 by Dover Publications Inc., New York).

30. Karon BS, Boyd JC, Klee GG. Glucose meter performance criteria for tight glycemic control estimated by simulation modeling. Clin Chem 2010;56:1091-7.

31. Ricos C, Alvarez V, Cava F, Garcia-Lario JV, Hernandez A, Jimenez CV, et al. Current databases on biologic variation: pros, cons and progress. Scand J Clin Lab Invest 1999;59:491-500.

32. Van den Berghe G, Wouters PJ, Bouillon R, Weekers F, Verwaest $C$, Schetz $M$, et al. Outcome benefit of intensive insulin therapy in the critically ill: insulin dose versus glycemic control. Crit Care Med 2003;31:359-66.

33. De Koninck AS, De Decker K, Van Bocxlaer J, Meeus P, Van Hoovels L. Analytical performance evaluation of four cartridge-type blood gas analyzers. Clin Chem Lab Med 2012;50:1083-91.

34. The Royal College of Pathologists of Australasia (RCPA). Chemical pathology quality assurance program. Available from: http://www.rcpaqap.com.au/chempath/gendocs/ uploadedfiles/Allowable-Limits.pdf. Accessed 25 January, 2013.

35. Slater-MacLean L, Cembrowski G, Chin D, Shalapay C, Binette T, Hegadoren K, et al. Accuracy of glycemic measurements in the critically ill. Diabetes Technol Ther 2008;10:169-77.

36. Vlasselaers D, Herpe TV, Milants I, Eerdekens M, Wouters PJ, Moor BD, et al. Blood glucose measurements in arterial blood of intensive care unit patients submitted to tight glycemic control: agreement between bedside tests. J Diabetes Sci Technol 2008;2:932-8.

37. Atkin SH, Dasmahapatra A, Jaker MA, Chorost MI, Reddy S. Fingerstick glucose determination in shock. Ann Intern Med 1991;114:1020-4.

38. Haupt A, Berg B, Paschen P, Dreyer M, Haring HU, Smedegaard J, et al. The effects of skin temperature and testing site on blood 
glucose measurements taken by a modern blood glucose monitoring device. Diabetes Technol Ther 2005;7:597-601.

39. US Food and Drug Administration (FDA). Review criteria assessment of portable blood glucose monitoring in vitro diagnostic devices using glucose oxidase, dehydrogenase or hexokinase methodology. Available from: http://www.fda.gov/
MedicalDevices/DeviceRegulationandGuidance/ GuidanceDocuments/ucm094134.htm. Accessed 25 January, 2013.

40. Karon BS, Scott R, Burritt MF, Santrach PJ. Comparison of lactate values between point-of-care and central laboratory analyzers. Am J Clin Pathol 2007;128:168-71. 